\title{
Hamstring Stiffness Returns More Rapidly After Static Stretching Than Range of Motion, Stretch Tolerance, and Isometric Peak Torque
}

\author{
Genki Hatano, Shigeyuki Suzuki, Shingo Matsuo, Satoshi Kataura, Kazuaki Yokoi, Taizan Fukaya, \\ Mitsuhiro Fujiwara, Yuji Asai, and Masahiro Iwata
}

\begin{abstract}
Context: Hamstring injuries are common, and lack of hamstring flexibility may predispose to injury. Static stretching not only increases range of motion (ROM) but also results in reduced muscle strength after stretching. The effects of stretching on the hamstring muscles and the duration of these effects remain unclear. Objective: To determine the effects of static stretching on the hamstrings and the duration of these effects. Design: Randomized crossover study. Setting: University laboratory. Participants: A total of 24 healthy volunteers. Interventions: The torque-angle relationship (ROM, passive torque [PT] at the onset of pain, and passive stiffness) and isometric muscle force using an isokinetic dynamometer were measured. After a 60-minute rest, the ROM of the dynamometer was set at the maximum tolerable intensity; this position was maintained for 300 seconds, while static PT was measured continuously. The torque-angle relationship and isometric muscle force after rest periods of 10, 20, and 30 minutes were remeasured. Main Outcome Measures: Change in static PT during stretching and changes in ROM, PT at the onset of pain, passive stiffness, and isometric muscle force before stretching were compared with 10, 20, and 30 minutes after stretching. Results: Static PT decreased significantly during stretching. Passive stiffness decreased significantly 10 and 20 minutes after stretching, but there was no significant prestretching versus poststretching difference after 30 minutes. PT at the onset of pain and ROM increased significantly after stretching at all rest intervals, while isometric muscle force decreased significantly after all rest intervals. Conclusions: The effect of static stretching on passive stiffness of the hamstrings was not maintained as long as the changes in ROM, stretch tolerance, and isometric muscle force. Therefore, frequent stretching is necessary to improve the viscoelasticity of the muscle-tendon unit. Muscle force decreased for 30 minutes after stretching; this should be considered prior to activities requiring maximal muscle strength.
\end{abstract}

Keywords: retention time, muscle stretching, flexibility, muscle force

Hamstring strain injuries are the most common noncontact injuries in Australian football, soccer, rugby, track and field, and American football. ${ }^{1}$ The injury rate for hamstring muscles is between $22 \%$ and $34 \%,{ }^{2}$ with a reinjury rate of $50 \%$ within 1 month. ${ }^{3}$ Sports activities that can cause injury to the hamstring muscles usually involve rapid acceleration and fast running. The eccentric contraction of the hamstrings to decelerate knee extension during the late swing phase of running activities is associated with such injuries. ${ }^{2,3}$ Lack of hamstring flexibility may also result in major muscle imbalances, which predisposes to muscle injuries, ${ }^{4}$ patellar tendinopathy, and patellofemoral pain, ${ }^{5}$ and facilitates the development of low back pain. ${ }^{6}$ Therefore, obtaining detailed information about the effects of stretching on the hamstring muscles is an important issue that could lead to an increase in the effectiveness of stretching, as the hamstrings are the most frequently stretched muscle group, in an attempt to prevent injury. ${ }^{7}$

Hatano is with the Institute of Sport Science, ASICS Corporation, Kobe, Japan. Suzuki and Matsuo are with Program in Physical and Occupational Therapy, Graduate School of Medicine, Nagoya University, Nagoya, Japan. Matsuo, Asai, and Iwata are with the Department of Rehabilitation, Faculty of Health Sciences, Nihon Fukushi University, Handa, Japan. Kataura is with Kobe Century Memorial Hospital, Kobe, Japan. Yokoi is with Kameda Medical Center, Kamogawa, Japan. Fukaya is with Kyoto Kujo Hospital, Kyoto, Japan. Fujiwara is with Kamiiida Rehabilitation Hospital, Nagoya, Japan. Iwata is with the Department of Physiology, University of Kentucky College of Medicine, Lexington, KY, USA. Iwata (iwata-m@n-fukushi.ac.jp) is corresponding author.
Flexibility and maximum joint range of motion (ROM) are important functional parameters in sport performance and rehabilitation that may affect the risk for muscle strain injury ${ }^{4}$ and influence the capacity to perform activities of daily living. ${ }^{8}$ Both of these parameters are compromised with aging ${ }^{9}$ and disease. ${ }^{10}$ The ability to move comfortably through a large ROM determines the successful performance of athletic tasks. Stretching is one such technique commonly used to acutely increase ROM. ${ }^{11-14}$ The proposed mechanisms for this include an increased stretch tolerance due to altered sensation ${ }^{12,14,15}$ and a change in the stiffness/ viscoelastic properties of the muscle-tendon unit, ${ }^{12-14,16-18}$ which result in improved movement and function. ${ }^{8,14}$

Injury prevention techniques commonly used in sports include proprioceptive neuromuscular facilitation, and ballistic, dynamic, and static stretching. However, static stretching remains the most widely used strategy, as it is relatively easy to perform, does not require excessive time or effort, and has a low risk of injuries. When static stretching is performed for the purpose of gaining flexibility, which is defined as the ability to move a joint through its complete ROM, ${ }^{19}$ the positive effects include increased maximum joint ROM, ${ }^{11-14}$ reduced resistance during stretching as assessed by static passive torque (SPT), ${ }^{13,14,16,20}$ and reduced stiffness of muscle-tendon units. ${ }^{12-14,16-18}$ These effects are thought to contribute to the prevention of injury. ${ }^{7}$ However, a negative effect of static stretching is reduced muscle strength after stretching, ${ }^{13,21-24}$ so athletes may avoid stretching prior to events that require maximal muscle strength performance. ${ }^{23,25}$ 
Although numerous studies have investigated the effects of static stretching, the duration of these effects remains unknown. The duration of the effects of stretching may range from several minutes to several hours, as measured by changes in the majority of performance indices for passive stiffness, ROM, and muscle strength. ${ }^{11,12,16,18,21,22,25}$ However, previous studies have involved different stretching times, intensities, and target muscles, so it is difficult to make simple comparisons of their results. Although Mizuno et $\mathrm{al}^{12,26,27}$ have studied the effects of acute static stretching on a number of variables and their retention time in the gastrocnemius muscle, to our knowledge, no studies have investigated multiple indices simultaneously and in detail in the hamstring muscles. Therefore, the duration of the effects of static stretching was investigated by comparing SPT, ROM, passive torque (PT) at the onset of pain, passive stiffness, and isometric muscle force before stretching versus 10, 20, and 30 minutes after stretching. The purpose of this study was to elucidate the duration of stretchinduced effects on the hamstrings to help guide more effective stretching techniques and to hypothesize that SPT, passive stiffness, and muscle force would be decreased, and PT at the onset of pain and ROM would be increased by stretching.

\section{Methods}

\section{Study Design}

A randomized crossover design was used to clarify the time course and progression of the stiffness of the hamstrings, and the ROM, stretch tolerance, and isometric peak torque of the knee flexors after static stretching of the hamstrings. The experiment was performed in Nihon Fukushi University laboratory, where the room temperature was maintained at $26^{\circ} \mathrm{C}$. The subjects each underwent stretching followed by either 10,20 , or 30 minutes of rest prior to measurements being taken to investigate the time course and progression of the effects of stretching. The order of the rest duration after stretching for each participant was determined randomly using a random number table, with participants selecting a number from 1 to 6 with different rest intervals each being assigned a number. Participants visited the laboratory on 4 occasions, with each visit separated by more than 24 hours. The first visit was a familiarization trial and the subsequent 3 visits were experimental trials. Participants completed all experimental trials within a 2-week time period. The criterion measures consisted of SPT, ROM of passive knee extension, PT at the onset of pain, passive stiffness, and isometric muscle force. All measurements except SPT were taken 60 minutes before static stretching and at specific rest periods after static stretching. SPT was measured during stretching. Each stretching session was performed at a similar time of day for each participant. Changes in the dependent variables before and after static stretching were compared between rest durations to investigate the retention time of the effect of static stretching.

\section{Participants}

A total of 24 healthy university students (11 males and 13 females) voluntarily participated in this study after being informed of the study purpose and protocol, and all participants provided written informed consent. This study was approved by the ethics committee for research on human subjects at Nihon Fukushi University (approval 4 number 11-07) and the human research ethics committee of Nagoya university (approval number 11-510), and
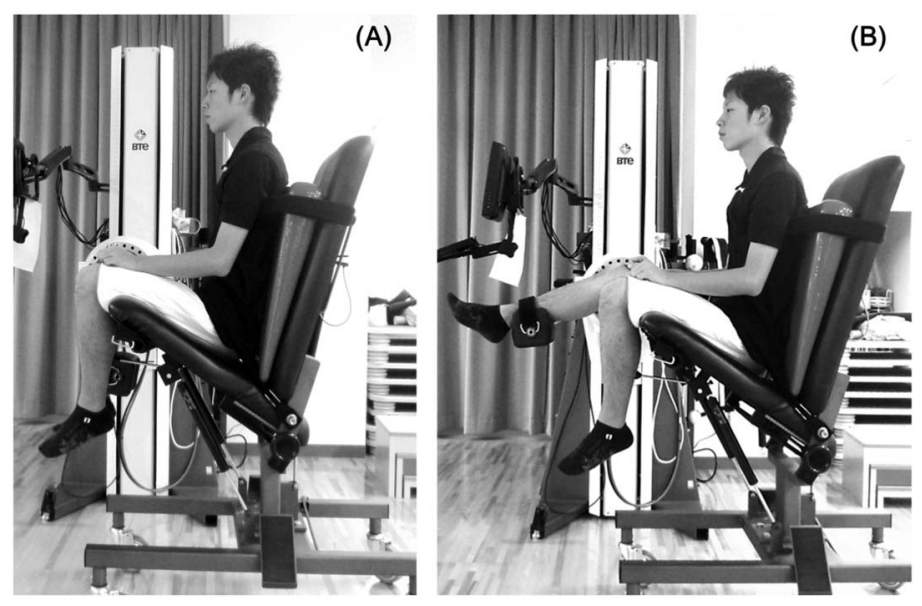

Figure 1 - Positioning for static stretching of the knee flexors before (A) and during stretching (B) as seen from the left side. (A) The seat of the dynamometer was maximally raised, and a wedge-shaped cushion was inserted between the trunk and backrest, creating a seat face-back angle of approximately $60^{\circ}$. The subjects were seated in this position and their chest, pelvis, and right thigh were stabilized with Velcro straps. The knee joint was aligned with the axis of the isokinetic dynamometer. The lever arm attachment was placed just proximal to the medial malleolus and stabilized with Velcro straps. (B) Subjects' right hamstrings were stretched using an isokinetic dynamometer. The stretch was stopped at tolerable intensity without pain.

complied with the requirements of the Declaration of Helsinki. The respective mean (SD) for age, height, body mass, and body mass index were $20.5(1.1)$ years, $163.4(8.8) \mathrm{cm}, 55.6(7.6) \mathrm{kg}$, and $20.8(2.0) \mathrm{kg} / \mathrm{m}^{2}$, respectively. The exclusion criteria included the following: history of hip or lower-extremity joint surgery; lowerextremity neurological findings, such as sensory impairment; those who were able to fully extend their knee from the measuring start position described below (see Figure 1); subjects taking medication that affected the muscles and/or hormones (including nonsteroidal anti-inflammatory drugs, statins, fluoroquinolones, and corticosteroids); and those who were engaged in competitive sports. Participants were instructed to maintain their normal dietary habits and to refrain from vigorous physical activity for 1 day before the experiment.

\section{Procedures}

Sitting Position. An isokinetic dynamometer (Primus RS; BTE Technologies, Corp, Hanover, MD) was used for testing. To enable the easier extension of the hamstrings, a measuring start position for the limbs was established using the methods described previously (Figure 1A). ${ }^{13,14}$ The seat of the apparatus was shifted to its maximum forward position ( $35^{\circ}$ from the horizontal position), and a wedge-shaped cushion was placed between the subject's back and the backrest of the seat so that the angle between the seat and backrest was approximately $60^{\circ}$. The subject's chest, pelvis, and right thigh were held in place using Velcro straps. The knee joint was aligned with the axis of rotation of the isokinetic dynamometer, and the lever arm attachment was placed proximal to the medial malleolus and stabilized with Velcro straps. Stretching and assessment of PT and isometric muscle force were performed in this position, with the mean angles of hip and knee flexion recorded as $111.8^{\circ}\left(2.8^{\circ}\right)$ and $111.1^{\circ}\left(2.6^{\circ}\right)$, respectively. 


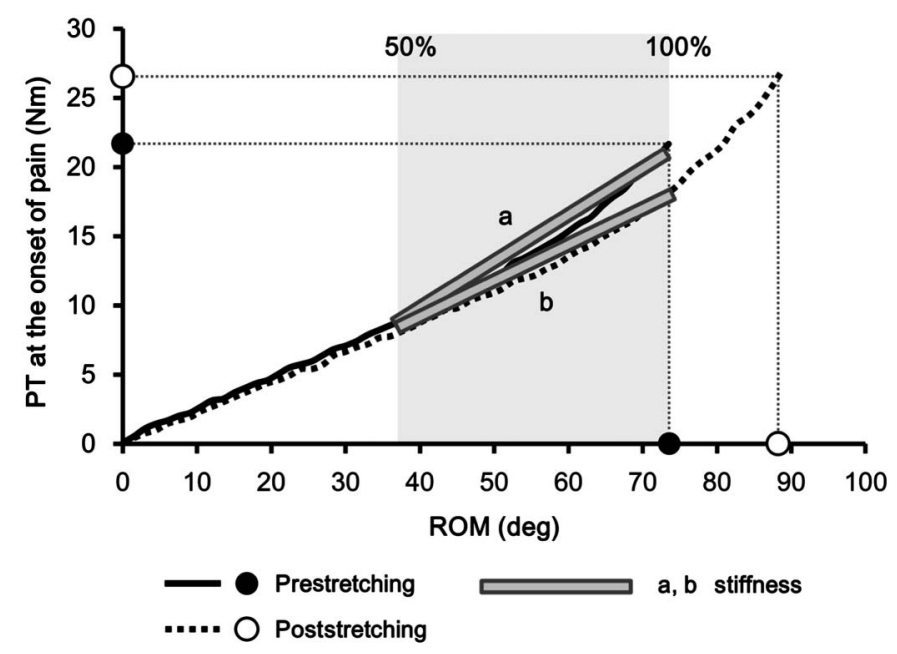

Figure 2 - Typical torque-angle curves of a subject before (prestretching) and 10 minutes after stretching (poststretching). Stiffness was determined by a regression line between 50\% and $100 \%$ of the prestretching ROM-PT relationship. Stiffness changed from a (0.346) to $b(0.273)$ for this example. PT at the onset of pain and ROM were determined by PT and knee extension angle at the onset of pain. ROM indicates range of motion; PT, passive torque.

Static Stretching. The right hamstring muscles were stretched using the isokinetic dynamometer from the start position shown in Figure 1 to the point of maximal extension while the angle of the hip joint remained constant. The ROM of the dynamometer was set at maximum tolerable intensity without stretching pain (participants were asked to stretch as much as possible but to stop short of feeling any pain; Figure 1B), and this position was maintained for 300 seconds. The joint angle was kept constant during the stretch. In this study, 300 seconds of static stretching was used, as a previous study showed that this length of static stretching at a tolerable intensity without pain significantly increased ROM and decreased passive stiffness and isometric force immediately after stretching. ${ }^{13}$ A familiarization session was performed at least 1 day before the first testing day, in which participants experienced all measurements and static stretching. The ROM was determined at each session.

The torque-angle relationship (ROM, PT at the onset of pain, and passive stiffness) was measured during a maximum ROM test (Figure 2) before maximal isometric muscle force was measured. On each of the 3 testing days, participants rested for 60 minutes to exclude the influence of the prestretching measurements. After the 60-minute rest, SPT was measured as the participants stretched their right hamstring muscles. After stretching, the torque-angle relationship (ROM, PT at the onset of pain, and passive stiffness) and isometric muscle force were measured after the specified rest periods in the same way as before stretching. The measurements were performed in this order for all participants, and all measurements for each participant were performed on a single day.

Dependent Variables. The dependent variables included SPT, ROM, PT at the onset of pain, passive stiffness, and isometric muscle force. SPT was measured during static stretching, whereas the other parameters were measured at the specified time points. The details of each measurement are provided below.

Static PT. The SPT (in newton meter) produced by the hamstrings during static stretching was measured continuously using the isokinetic dynamometer, and the torque signal was transferred to a PowerLab system (ADInstruments, NSW, Australia) and stored on a PC (Dynabook Satellite J50; Toshiba, Tokyo, Japan) for subsequent analyses. To assess the magnitude of "stress relaxation" 16,19 that occurred during stretching, the changes in SPT from the onset of stretching $(0 \mathrm{~s})$ to the end of stretching (300 s) were calculated using LabChart software (LabChart 4; ADInstruments) based on previous research. ${ }^{13,14}$

ROM, PT at the Onset of Pain, and Passive Stiffness. ROM, PT at the onset of pain, and passive stiffness were calculated from the torque-angle curve obtained using the isokinetic dynamometer, and the torque and angle signals were analog-digital converted and stored on a PC for analyses. Although each participant was sitting in the chair (Figure 1A), the knee was extended passively at $5^{\circ}$ per second to the point of maximum knee extension just before the subjective onset of pain (if discomfort or pain was felt, the extension was decreased slightly to a point where the subject no longer felt discomfort), and the torque was recorded continuously to obtain the torque-angle relationship during passive knee extension. ${ }^{13,14}$ ROM (in degrees) was defined as the maximum knee extension angle from the initial position $\left(0^{\circ}\right)$, and PT at the onset of pain (in newton meter) was defined as the torque at the onset of pain. ${ }^{12,14}$ Passive stiffness (in newton meter per degree) was defined as the slope of the regression line that was calculated from the torque-angle relationship using the least-squares method. ${ }^{13,14}$ Stiffness was calculated from the same-knee extension angle range before and after stretching, and the prestretching value was compared with that after stretching. The calculated knee extension angle range was defined as the angle from the 50\% maximum knee extension angle to the prestretching maximum knee extension angle. However, if the poststretching maximum knee extension angle was smaller than that before stretching, the stiffness before and after stretching was calculated from the 50\% maximum knee extension angle to the poststretching maximum knee extension angle.

Isometric Muscle Force. Isometric muscle force (in newton meter) was measured in the same position as that used for the starting point of the static stretch (Figure 1A), which was approximately 110 degrees of flexion at the knee. ${ }^{13,14}$ The participants were instructed to generate a maximum knee flexion force for 6 seconds with their arms crossed in front of their chest. Verbal encouragement was provided during the measurement. Peak torque was recorded. Only 1 test was performed so as not to fatigue the participants.

\section{Measurement Reliability}

The test-retest reliability for all dependent variables was determined in 5 males and 1 female (mean age $=21.7$ [1.6] y) before the data collection in this study. The 2 tests were separated by 1 to 7 days and were performed at the same time of the day $( \pm 1 \mathrm{~h})$. The intraclass correlation coefficients revealed high reliability for all measures (SPT: 0.80; ROM: 0.84; PT at the onset of pain: 0.89; passive stiffness: 0.91; and isometric muscle force: 0.88). The coefficient of variation for the measures also showed acceptable reliability (SPT: 7.9\%; ROM: 3.7\%; PT at the onset of pain: 7.4\%; passive stiffness: $9.2 \%$; and isometric muscle force: $3.8 \%$ ).

\section{Statistical Analysis}

The Shapiro-Wilk test was used to assess the normality of the measured values. As some of the dependent variables were not 
Table 1 Change in SPT From the Onset to the End of Stretching

\begin{tabular}{|c|c|c|c|c|c|}
\hline & & Onset & End & & Difference, mean (SD) \\
\hline Outcome & Condition & $\overline{\text { Mean (SD) }}$ & Mean (SD) & $P$ value & $(95 \% \mathrm{Cl})$ \\
\hline \multirow{6}{*}{$\mathrm{SPT}$, in $\mathrm{N} \cdot \mathrm{m}$} & 10-min rest & $22.0(8.3)$ & $19.8(7.0)^{*}$ & $<.001$ & $-2.2(1.9)$ \\
\hline & & & & & $(-3.0$ to -1.4$)$ \\
\hline & 20-min rest & $22.5(8.1)$ & $19.9(6.8)^{*}$ & $<.001$ & $-2.6(1.8)$ \\
\hline & & & & & $(-3.3$ to -1.8$)$ \\
\hline & 30-min rest & $22.1(7.4)$ & $19.7(6.0)^{*}$ & $<.001$ & $-2.4(1.6)$ \\
\hline & & & & & $(-3.1$ to -1.8$)$ \\
\hline
\end{tabular}

Abbreviation: SPT, static passive torque.

${ }^{\mathrm{a}}$ Difference $=$ End value - Onset value.

$* P<.05$ compared with the onset value.

normally distributed, nonparametric tests were used for all variables. The Wilcoxon signed-rank test was performed to compare the absolute prestretching and poststretching values. The Friedman test was used to compare the difference between the variables for the different durations of rest after stretching (eg, the 10-, 20-, and 30 -min prestretch vs the 10-, 20-, and 30-min poststretch). When a significant difference was found, a Bonferroni post hoc test was used to check for significant differences between the rest intervals. The coefficient of variation for all participants was also calculated from the average value and $\mathrm{SD}$ of the prestretching values at each rest interval (10, 20, and $30 \mathrm{~min}$ ). SPSS (version 16.0J; SPSS Japan Inc, Tokyo, Japan) was used for the analyses, and the significance was set at $P<.05$. All data are expressed as mean (SD).

\section{Results}

The SPT results used as the confirmation index are shown in Table 1 . The SPT significantly decreased from the prestretch value at all rest time points, and there were no differences in the change in SPT between the 10-, 20-, and 30-minute rest time points. All subjects were able to perform and complete the prescribed stretching exercises.

The results of passive stiffness, PT at the onset of pain, ROM, and isometric muscle force are shown in Table 2. Passive stiffness significantly decreased in the 10- and 20-minute rest interval after stretching, but there was no significant prestretching versus poststretching difference in the 30-minute rest interval after stretching. PT at the onset of pain and ROM significantly increased after stretching at all rest intervals, whereas isometric muscle force significantly decreased after stretching at all rest intervals. Furthermore, there were no differences between the prestretching and poststretching values (10- vs 20 - vs 30 -min rest intervals after stretching) for any of the dependent variables when assessed using the Friedman test $(P>.05)$.

The coefficient of variation for the prestretching values at each rest interval $(10,20$, and $30 \mathrm{~min})$ revealed that the reliability was acceptable for all measures (SPT: 8.7\%; ROM: 3.4\%; PT at the onset of pain: $6.7 \%$; passive stiffness: $9.4 \%$; and isometric muscle force: $5.7 \%$ ) between different rest periods.

\section{Discussion}

The duration of the effects of stretching was compared and investigated on a variety of performance indices by measuring the passive stiffness, PT at the onset of pain, ROM, and isometric muscle force of the hamstrings of healthy subjects before stretching and at 10, 20, and 30 minutes after stretching. The SPT, which was used as the confirmation index, significantly decreased after stretching at all rest periods, which is consistent with previous results. ${ }^{13,14,16,20}$ Factors that contribute to the decrease in SPT include (afferent type Ib sensory nerve fiber) inhibition during stretching that decreases the excitability of ventral horn cells, other neurophysiological factors, ${ }^{28}$ and the stress relaxation ${ }^{16,20}$ that occurs due to the viscoelasticity of the muscle-tendon unit and other kinetic factors. The absence of differences in the degree of change in SPT confirms that an equal amount of stretching load was applied in all participants in each stretching session. Hence, it was determined that it is possible to compare the results of the other performance indices at the different rest intervals.

The effects of static stretching of the hamstrings for 300 seconds lasted between 20 and 30 minutes on passive stiffness and for 30 or more minutes regarding PT at the onset of pain, ROM, and isometric muscle force. Specifically, the most important finding was that after static stretching of the hamstrings, passive stiffness returns earlier than does ROM, PT at the onset of pain, and isometric muscle force.

Passive stiffness significantly declined after stretching at the 10- and 20-minute rest intervals. Passive stiffness, which is calculated from the torque-angle curve, is thought to reflect the viscoelasticity of the muscle-tendon unit, ${ }^{16}$ and the effects of static stretching on the passive stiffness of the gastrocnemius muscle reportedly last for 15 to 20 minutes. $^{12,18}$ However, although Magnusson et $\mathrm{al}^{16}$ found that passive stiffness declined immediately after stretching of the hamstrings, the effects disappeared 60 minutes after stretching, and they did not provide any details on the duration of the effects. Mizuno et al ${ }^{12,26}$ reported that the duration of the effect of stretching on the passive stiffness of the gastrocnemius muscle was shorter than the duration of the effect on PT at the onset of pain and ROM. Notably, although they had similar results to our study regarding increases in ROM and PT after stretching, they had a recovery of stiffness within 5 to 15 minutes after stretching ${ }^{12,26}$ and a recovery of maximal voluntary contraction torque within 10 minutes after stretching. ${ }^{27} \mathrm{~A}$ simple comparison is difficult because their studies focused on a muscle with different morphology and muscle fiber composition, and they also had a different protocol for taking measurements after stretching. They measured maximal voluntary contraction torque multiple times in the first 15 minutes after stretching, so this may have enhanced the recovery of the stretching-induced decreases in maximal voluntary contraction torque. ${ }^{27}$ However, the present results indicate that the 2 muscle groups have similar results, albeit 
Table 2 Changes in Passive Stiffness, PT at the Onset of Pain, ROM, and Isometric Muscle Force Before and After Static Stretching Under Each Condition

\begin{tabular}{|c|c|c|c|c|c|}
\hline \multirow[b]{2}{*}{ Outcome } & \multirow[b]{2}{*}{ Condition } & \multirow{2}{*}{$\frac{\text { PRE }}{\text { Mean (SD) }}$} & \multirow{2}{*}{$\begin{array}{c}\text { POST } \\
\text { Mean (SD) }\end{array}$} & \multirow[b]{2}{*}{$P$ value } & \multirow{2}{*}{$\frac{\text { Difference, mean (SD) }^{a}}{(95 \% \mathrm{Cl})}$} \\
\hline & & & & & \\
\hline \multirow[t]{6}{*}{ Passive stiffness, in $\mathrm{N} \cdot \mathrm{m} / \mathrm{deg}$} & 10-min rest & $0.367(0.133)$ & $0.342(0.116)^{*}$ & .005 & $-0.025(0.052)$ \\
\hline & & & & & $(-0.047$ to -0.003$)$ \\
\hline & 20-min rest & $0.373(0.138)$ & $0.349(0.127)^{*}$ & .007 & $-0.024(0.047)$ \\
\hline & & & & & $(-0.043$ to -0.003$)$ \\
\hline & 30-min rest & $0.357(0.483)$ & $0.338(0.131)$ & .136 & $-0.019(0.057)$ \\
\hline & & & & & $(-0.043$ to 0.005$)$ \\
\hline \multirow[t]{6}{*}{ PT at the onset of pain, in $\mathrm{N} \cdot \mathrm{m}$} & 10-min rest & $25.5(10.0)$ & $27.7(10.6)^{*}$ & .002 & $2.2(2.6)$ \\
\hline & & & & & (1.1 to 3.3$)$ \\
\hline & 20-min rest & $25.5(10.2)$ & $28.0(11.3)^{*}$ & .001 & $2.5(3.0)$ \\
\hline & & & & & (1.3 to 3.8$)$ \\
\hline & 30-min rest & $24.7(10.3)$ & $26.8(10.5)^{*}$ & .004 & $2.1(2.3)$ \\
\hline & & & & & (1.1 to 3.0$)$ \\
\hline \multirow[t]{6}{*}{$\mathrm{ROM}$, in deg } & 10-min rest & $77.0(10.5)$ & $82.9(10.7)^{*}$ & $<.001$ & $5.9(3.9)$ \\
\hline & & & & & (4.2 to 7.5$)$ \\
\hline & 20-min rest & $77.2(9.1)$ & $83.0(10.4)^{*}$ & $<.001$ & $5.8(2.7)$ \\
\hline & & & & & (4.7 to 7.0$)$ \\
\hline & 30-min rest & $77.8(8.9)$ & $82.8(9.8)^{*}$ & $<.001$ & $4.9(2.5)$ \\
\hline & & & & & (3.9 to 6.0 ) \\
\hline \multirow[t]{6}{*}{ Isometric muscle force, in $\mathrm{N} \cdot \mathrm{m}$} & 10-min rest & $60.1(17.3)$ & $57.6(16.1)^{*}$ & .017 & $-2.5(5.3)$ \\
\hline & & & & & $(-4.8$ to -0.3$)$ \\
\hline & 20-min rest & $59.3(17.2)$ & $57.1(15.6)^{*}$ & .033 & $-2.2(3.5)$ \\
\hline & & & & & $(-3.6$ to -0.7$)$ \\
\hline & 30-min rest & $58.0(17.7)$ & $56.1(16.5)^{*}$ & .006 & $-1.8(3.8)$ \\
\hline & & & & & $(-3.4$ to -0.2$)$ \\
\hline
\end{tabular}

Abbreviations: POST, poststretching; PRE, prestretching; PT, passive torque; ROM, range of motion.

${ }^{\text {a Difference }}=$ POST value - PRE value.

$* P<.05$ compared with the PRE value.

with a different rate of recovery of these variables. Furthermore, previous studies that involved stretching the hamstrings for relatively short periods of time reported no changes in passive stiffness. ${ }^{29,30}$ However, passive stiffness decreases when the stretching time is longer, ${ }^{13,16,17}$ suggesting that changes in stiffness require longer periods of extension than the other indices. Ryan et $\mathrm{al}^{18}$ reported that longer stretching times result in longer maintenance of decrease in passive stiffness and that viscoelastic recoil (which causes the muscle to tend to return to its original shape because of the viscoelasticity of the tissues) was active in the early loss of effect. Based on the above results, the duration of the effect on passive stiffness was shorter because changes in passive stiffness due to stretching require more extension stimuli than the other indices, as the viscoelasticity of the tissues causes a tendency to return to their original shape.

Although the duration of the effect of stretching on passive stiffness was less than 30 minutes, the effect on ROM was maintained for at least 30 minutes. Factors that have been shown to contribute to increased ROM include decreased passive stiffness of the muscle-tendon unit and increased stretch tolerance. ${ }^{12}$ However, ROM reportedly still increases in cases in which passive stiffness does not decline. ${ }^{15}$ This indicates that the main factor related to increases in ROM is stretch tolerance. Stretch tolerance is thought to be the tolerance of the tensile strength produced in muscles that are subjected to passive extension. In previous studies, the index used to reflect this was PT at the onset of pain. ${ }^{12,14}$ In this study, PT was measured at the point immediately prior to the onset of pain in the posterior thigh. Hence, the PT at the onset of pain values indicates the resistance that occurred in the hamstring immediately prior to the onset of pain in the posterior thigh. As seen in previous studies, ${ }^{12,14}$ increases in the PT at the onset of pain after stretching indicate that stretch tolerance increased after stretching. However, much about the mechanism of stretch tolerance remains unknown, and this issue requires further study.

Isometric muscle force was maintained in a state of decline for at least 30 minutes after stretching, which is consistent with previous studies. ${ }^{13,21-24}$ Previous studies have reported declines in muscle force after static stretching for a duration of only several minutes, ${ }^{22}$ or up to 2 hours or more, ${ }^{24,25}$ which indicate that there is no current consensus. The degree of muscle force decline has been reported to be related to stretching time. ${ }^{23}$ In this study, which involved stretching for the relatively long duration of 5 minutes, declines in muscle force were maintained for at least 30 minutes after stretching. Factors related to decline in muscle force after stretching include changes in dynamic characteristics (ie, changes in the optimal muscle length during application of muscle force due to changes in the force-length relationship of the muscle-tendon unit, ${ }^{31}$ which could also potentially affect muscle stiffness), 
declines in the transmission efficiency of force due to changes in the viscoelasticity of the muscle-tendon unit, ${ }^{32}$ reduced mobilization of neuromuscular units, ${ }^{21}$ and excitation-contraction coupling. ${ }^{24}$ In addition, studies have reported that extensions of the electromechanical delay caused by the effect on excitationcontraction coupling during the period immediately after stretching to 15 minutes after stretching are synchronized, and that after 15 minutes, poststretching changes in the viscoelasticity and the force-length relationship of the main muscle-tendon unit have a major effect. ${ }^{21,24}$

It is assumed that the declines in isometric muscle force after stretching in this study were influenced by changes in the dynamic characteristics of the muscle-tendon unit and neurophysiological factors. It is also assumed that the effects of the neurophysiological factors after stretching declined over time, and changes in dynamic characteristics had a major effect. However, based on the passive stiffness results in this study, it is concluded that declines in the viscoelasticity of the muscle-tendon unit had very little effect on declines in muscle force, and therefore, it is possible that changes in the optimal muscle length had the greatest effect on declines in muscle force. Optimal muscle length may affect stiffness; but to our knowledge, this has not been directly studied and requires further elucidation. In addition, as the relevant mechanisms were not studied, further research is required to investigate the changes in dynamic and electrophysiological characteristics using electromyography, mechanomyography, electrostimulator devices, and diagnostic sonography.

This study found that changes in the passive stiffness of the hamstrings after static stretching were not maintained as long as the changes in ROM, stretch tolerance, and isometric muscle force. Therefore, as the effect on passive stiffness was maintained for a relatively shorter period of time, frequent stretching is necessary when aiming to improve the viscoelasticity of the muscle-tendon unit. In addition, the present results suggest that poststretching declines in muscle force may be maintained for as long as or longer than the effects on the other indices. Thus, poststretching decline in muscle force must be considered prior to engaging in activities that require maximal muscle strength.

This study had several limitations. First, all the participants were healthy university students, which limits the generalizability of the findings to other age groups. Second, the examiner performing the measurements was not blinded to the participants or the rest intervals. Third, only the acute effects of static stretching on passive stiffness, PT at the onset of pain, ROM, and isometric muscle force were examined. Finally, although recent literature has shown that female hormones may affect muscle stiffness, ${ }^{33}$ males and females were not compared, and both males and females were included in this study. As the prolonged effects were not examined after more than a few days or the effects of a stretching training program that lasts several weeks, the results may not apply to longterm stretching programs.

\section{Conclusions}

The duration of the effects of stretching for 300 seconds was investigated on the hamstrings by measuring passive stiffness, PT at the onset of pain, ROM, and isometric muscle force after rest periods of 10,20 , and 30 minutes. The duration of the poststretching effect on PT at the onset of pain, ROM, and isometric muscle force was at least 30 minutes, whereas the duration of the poststretching effect on passive stiffness was less than 30 minutes.

\section{Acknowledgments}

This work was supported in part by JSPS KAKENHI (grant numbers 26750288 [S.M.] and 16K01503 [S.S.]) from the Japan Society for the Promotion of Science (JSPS), and in part by a grant from the Public Advertisement Research Project of Nihon Fukushi University.

\section{References}

1. Opar DA, Williams MD, Shield AJ. Hamstring strain injuries: factors that lead to injury and re-injury. Sports Med. 2012;42:209226. PubMed ID: 22239734 doi:10.2165/11594800-00000000000000

2. Orchard J, Seward H. Epidemiology of injuries in the Australian Football League, seasons 1997-2000. Br J Sports Med. 2002;36: 39-44.

3. Brooks JH, Fuller CW, Kemp SP, Reddin DB. Incidence, risk, and prevention of hamstring muscle injuries in professional rugby union. Am J Sports Med. 2006;34:1297-1306. PubMed ID: 16493170 doi: $10.1177 / 0363546505286022$

4. Witvrouw E, Danneels L, Asselman P, D'Have T, Cambier D. Muscle flexibility as a risk factor for developing muscle injuries in male professional soccer players. A prospective study. Am J Sports Med. 2003;31:41-46. PubMed ID: 12531755 doi:10.1177/ 03635465030310011801

5. Smith AD, Stroud L, McQueen C. Flexibility and anterior knee pain in adolescent elite figure skaters. J Pediatr Orthop. 1991;11:77-82. PubMed ID: 1988483 doi:10.1097/01241398-199101000-00015

6. Sadler SG, Spink MJ, Ho A, De Jonge XJ, Chuter VH. Restriction in lateral bending range of motion, lumbar lordosis, and hamstring flexibility predicts the development of low back pain: a systematic review of prospective cohort studies. BMC Musculoskelet Disord. 2017;18:179. PubMed ID: 28476110 doi:10.1186/s12891017-1534-0

7. Malliaropoulos N, Papalexandris S, Papalada A, Papacostas E. The role of stretching in rehabilitation of hamstring injuries: 80 athletes follow-up. Med Sci Sports Exerc. 2004;36:756-759. PubMed ID: 15126706 doi:10.1249/01.MSS.0000126393.20025.5E

8. Rodacki AL, Souza RM, Ugrinowitsch C, Cristopoliski F, Fowler NE. Transient effects of stretching exercises on gait parameters of elderly women. Man Ther. 2009;14:167-172. PubMed ID: 18314375 doi:10.1016/j.math.2008.01.006

9. Holland GJ, Tanaka K, Shigematsu R, Nakagaichi M. Flexibility and physical functions of older adults: a review. J Aging Phys Activ. 2002;10:169-206. doi:10.1123/japa.10.2.169

10. Duffin AC, Donaghue KC, Potter M, et al. Limited joint mobility in the hands and feet of adolescents with type 1 diabetes mellitus. Diabet Med. 1999;16:125-130. PubMed ID: 10229305 doi:10.1046/j.14645491.1999.00030.x

11. Depino GM, Webright WG, Arnold BL. Duration of maintained hamstring flexibility after cessation of an acute static stretching protocol. J Athl Train. 2000;35:56-59. PubMed ID: 16558609

12. Mizuno T, Matsumoto M, Umemura Y. Viscoelasticity of the muscletendon unit is returned more rapidly than range of motion after stretching. Scand J Med Sci Sports. 2013;23:23-30. PubMed ID: 21564309 doi:10.1111/j.1600-0838.2011.01329.x

13. Matsuo S, Suzuki S, Iwata M, et al. Acute effects of different stretching durations on passive torque, mobility, and isometric muscle force. J Strength Cond Res. 2013;27:3367-3376. PubMed ID: 23524367 doi:10.1519/JSC.0b013e318290c26f

14. Matsuo S, Suzuki S, Iwata M, Hatano G, Nosaka K. Changes in force and stiffness after static stretching of eccentrically-damaged 
hamstrings. Eur J Appl Physiol. 2015;115:981-991. PubMed ID: 25526850 doi:10.1007/s00421-014-3079-3

15. Weppler $\mathrm{CH}$, Magnusson SP. Increasing muscle extensibility: a matter of increasing length or modifying sensation? Phys Ther. 2010;90:438-449. PubMed ID: 20075147 doi:10.2522/ptj.20090012

16. Magnusson SP, Simonsen EB, Aagaard P, Kjaer M. Biomechanical responses to repeated stretches in human hamstring muscle in vivo. Am J Sports Med. 1996;24:622-628. PubMed ID: 8883682 doi:10. 1177/036354659602400510

17. Nordez A, Cornu C, McNair P. Acute effects of static stretching on passive stiffness of the hamstring muscles calculated using different mathematical models. Clin Biomech. 2006;21:755-760. doi:10.1016/ j.clinbiomech.2006.03.005

18. Ryan ED, Beck TW, Herda TJ, et al. The time course of musculotendinous stiffness responses following different durations of passive stretching. J Orthop Sports Phys Ther. 2008;38:632-639. PubMed ID: 18827325 doi:10.2519/jospt.2008.2843

19. American College of Sports Medicine. ACSM's Guidelines for Exercise Testing and Prescription. 10th ed. Philadelphia, PA: Wolters Kluwer/Lippincott Williams \& Wilkins Health; 2017.

20. Magnusson SP, Simonsen EB, Dyhre-Poulsen P, Aagaard P, Mohr T, Kjaer M. Viscoelastic stress relaxation during static stretch in human skeletal muscle in the absence of EMG activity. Scand J Med Sci Sports. 1996;6:323-328. PubMed ID: 9046541 doi:10.1111/j.16000838.1996.tb00101.x

21. Fowles JR, Sale DG, MacDougall JD. Reduced strength after passive stretch of the human plantarflexors. J Appl Physiol. 2000;89:11791188. PubMed ID: 10956367 doi:10.1152/jappl.2000.89.3.1179

22. Weir DE, Tingley J, Elder GCB. Acute passive stretching alters the mechanical properties of human plantar flexors and the optimal angle for maximal voluntary contraction. Eur J Appl Physiol. 2005;93:614623. PubMed ID: 15578204 doi:10.1007/s00421-004-1265-4

23. Rubini EC, Costa ALL, Gomes PSC. The effects of stretching on strength performance. Sports Med. 2007;37:213-224. PubMed ID: 17326697 doi:10.2165/00007256-200737030-00003

24. Esposito F, Limonta E, Ce E. Passive stretching effects on electromechanical delay and time course of recovery in human skeletal muscle: new insights from an electromyographic and mechanomyographic combined approach. Eur J Appl Physiol. 2011;111:485-495. PubMed ID: 20886228 doi:10.1007/s00421-010-1659-4

25. Power K, Behm D, Cahill F, Carroll M, Young W. An acute bout of static stretching: effects on force and jumping performance. Med Sci Sports Exerc. 2004:36:1389-1396. PubMed ID: 15292748 doi:10. 1249/01.MSS.0000135775.51937.53

26. Mizuno T, Matsumoto M, Umemura Y. Decrements in stiffness are restored within 10 min. Int J Sports Med. 2013;34:484-490. PubMed ID: 23143704

27. Mizuno T, Matsumoto M, Umemura Y. Stretching-induced deficit of maximal isometric torque is restored within 10 minutes. J Strength Cond Res. 2014;28:147-153. PubMed ID: 23615480 doi:10.1519/ JSC.0b013e3182964220

28. Guissard N, Duchateau J, Hainaut K. Mechanisms of decreased motoneurone excitation during passive muscle stretching. Exp Brain Res. 2001;137:163-169. PubMed ID: 11315544 doi:10.1007/ s002210000648

29. Magnusson SP, Aagard P, Simonsen E, Bojsen-Møller F. A biomechanical evaluation of cyclic and static stretch in human skeletal muscle. Int J Sports Med. 1998;19:310-316. PubMed ID: 9721053 doi:10.1055/s-2007-971923

30. Magnusson SP, Aagaard P, Nielson JJ. Passive energy return after repeated stretches of the hamstring muscle-tendon unit. Med Sci Sports Exerc. 2000;32:1160-1164. PubMed ID: 10862546 doi:10. 1097/00005768-200006000-00020

31. McHugh MP, Nesse M. Effect of stretching on strength loss and pain after eccentric exercise. Med Sci Sports Exerc. 2008;40:566-573. PubMed ID: 18379222 doi:10.1249/MSS.0b013e31815d2f8c

32. Costa PB, Ryan ED, Herda TJ, Walter AA, Hoge KM, Cramer JT. Acute effects of passive stretching on the electromechanical delay and evoked twitch properties. Eur J Appl Physiol. 2010;108:301-310. PubMed ID: 19784666 doi:10.1007/s00421-009-1214-3

33. Bell DR, Blackburn JT, Norcorss MF, et al. Estrogen and muscle stiffness have a negative relationship in females. Knee Surg Sports Traumatol Arthrosc. 2012;20:361-367. PubMed ID: 21695466 doi:10.1007/s00167-011-1577-y 